\title{
Weyl and Zariski chambers on K3 surfaces
}

\author{
Thomas Bauer and Michael Funke
}

June 2, 2010

\begin{abstract}
The big cone of every K3 surface admits two natural chamber decompositions: the decomposition into Zariski chambers, and the decomposition into simple Weyl chambers. In the present paper we compare these two decompositions and we study their mutual relationship: First, we give a numerical criterion for the two decompositions to coincide. Secondly, we study the mutual inclusions of Zariski and simple Weyl chambers. Finally, we establish the fact that - even though the decompositions themselves may differ - the number of Zariski chambers always equals the number of simple Weyl chambers.
\end{abstract}

\section{Introduction}

The purpose of this note is to study two natural chamber decompositions of the big cone on K3 surfaces: On the one hand one has the decomposition into Zariski chambers, which is important when considering base loci and volumes of line bundles (see [BKS]), and on the other hand one has the well-known decomposition into simple Weyl chambers, which is given by the hyperplanes that are dual to the negative curves. We compare these two decompositions and we study their geometry - by determining the mutual inclusions of chambers and by asking for the number of chambers into which the big cone is divided.

For a more detailed description, consider a smooth projective variety $X$ of dimension $n$ and a divisor $D$ on $X$. One defines the volume of $D$ as the real number

$$
\operatorname{vol}_{X}(D) \stackrel{\text { def }}{=} \limsup _{k} \frac{h^{0}(X, k D)}{k^{n} / n !}
$$

and one calls $D$ big if its volume is positive. The big cone $\operatorname{Big}(X)$ is then the cone in the Néron-Severi vector space $\mathrm{NS}(X) \otimes \mathbb{R}$ that is generated by the big divisors. These have recently attracted a lot of attention, as it turned out that many geometric, cohomological, and numerical aspects of the picture that one has classically for ample line bundles extend to big divisors (see [Nak, Laz, ELMNP1, ELMNP2]). The main result of [BKS] states that on surfaces the big cone admits a locally finite decomposition into rational locally polyhedral subcones such that

- on each subcone the volume function is given by a single polynomial of degree two, and

- in the interior of each of the subcones the stable base loci are constant.

Both facts are explained by the variation of the Zariski decomposition of big divisors: In the interior of the subcones the support of the negative part of the Zariski decomposition is constant. These subcones have therefore been called Zariski chambers in $[\mathrm{BKS}$. 
Suppose now that $X$ is a K3 surface. In that case, an interesting second point of view is provided by the Weyl chamber decomposition. We consider here in particular simple Weyl chambers, which are defined by the intersection behaviour of big divisors with (-2)-curves (see Sect. 1 for details).

Given that there are two natural decompositions of the big cone of a K3 surface, it is an obvious task to compare them. In [BKS, Sect. 3.2] the erroneous claim was made that the two decompositions always coincide. They may however differ, depending on the geometry of the surface, and our first result gives the precise condition when this is the case:

Theorem 1. Let $X$ be a K3 surface. Then the following conditions are equivalent.

(i) The interiors of the Zariski chambers coincide with the simple Weyl chambers.

(ii) There is no pair of (-2)-curves $C_{1}, C_{2} \subset X$ such that

$$
C_{1} \cdot C_{2}=1 \text {. }
$$

There exist K3 surfaces such that (i) and (ii) hold, and there exist K3 surfaces where these conditions do not hold.

This theorem is a consequence of the following more general result about the geometry of Zariski and Weyl chambers. To state it, we will use the following notation: When $\mathcal{S}=\left\{C_{1}, \ldots, C_{r}\right\}$ is a set of $(-2)$-curves whose intersection matrix is negative definite, then $Z_{\mathcal{S}}$ will denote the Zariski chamber consisting of the big divisors whose negative part is supported on $C_{1} \cup \ldots \cup C_{r}$, and $W_{\mathcal{S}}$ will denote the simple Weyl chamber consisting of the divisors that intersect $C_{1}, \ldots, C_{r}$ negatively and all other $(-2)$-curves positively (see Sect. 1 for the details). Furthermore, we will write $\stackrel{\circ}{Z}_{S}$ to denote the interior of the chamber $Z_{S}$.

Theorem 2. Let $X$ be a $K 3$ surface, and let $\mathcal{S}$ be a set of $(-2)$-curves on $X$ whose intersection matrix is negative definite.

(i) We have $W_{\mathcal{S}} \subset Z_{\mathcal{S}}$ if and only if the following condition holds: If $C^{\prime}$ is a curve with $C^{\prime} \notin \mathcal{S}$ such that the intersection matrix of the set $\mathcal{S} \cup\left\{C^{\prime}\right\}$ is negative definite, then $C^{\prime} \cdot C=0$ for all $C \in \mathcal{S}$.

(ii) We have $\stackrel{\circ}{Z}_{\mathcal{S}} \subset W_{\mathcal{S}}$ if and only if $C_{1} \cdot C_{2} \neq 1$ for all curves $C_{1}, C_{2} \subset S$.

We will study in Sect. 3 in detail examples of K3 surfaces where various cases of inclusions and equalities of chambers occur.

Given the fact that the decompositions may well be different, it is nice and somewhat surprising that on any K3 surface there are precisely as many Zariski chambers as there are Weyl chambers:

Theorem 3. Let $X$ be a K3 surface. Then there is a canonical bijection between the set of Zariski chambers in $\operatorname{Big}(X)$ and the set of simple Weyl chambers in $\operatorname{Big}(X)$.

\section{Chamber decompositions}

Zariski chambers. We recall very briefly the Zariski chamber decomposition from [BKS]. Let $X$ be a smooth projective surface over $\mathbb{C}$. To any big and nef $\mathbb{R}$-divisor $P$, one associates the Zariski chamber $\Sigma_{P}$, which by definition consists of 
all divisors in $\operatorname{Big}(X)$ such that the irreducible curves in the negative part of the Zariski decomposition of $D$ are precisely the curves $C$ with $P \cdot C=0$. One has by [BKS, Lemma 1.6]:

For two big and nef divisors $P$ and $P^{\prime}$, the Zariski chambers $\Sigma_{P}$ and $\Sigma_{P^{\prime}}$ are either equal or disjoint. The Zariski chambers yield a decomposition of the big cone.

If $H$ is an ample divisor, then the interior of the chamber $\Sigma_{H}$ is the ample cone, its closure is the nef cone. (Note that $\Sigma_{H}$ itself need not be open or closed.) By way of abbreviation, we will refer to this chamber as the nef chamber in the sequel. We will make frequent use of the following basic observation.

Lemma 1.1. The set of Zariski chambers on a smooth projective surface $X$ that are different from the nef chamber is in bijective correspondence with the set of reduced divisors on $X$ whose intersection matrix is negative definite.

Proof. The statement is Proposition 1.1 from [BFN]. For the benefit of the reader, we briefly give the argument. For a chamber $\Sigma_{P}$ we consider the irreducible curves $C_{1}, \ldots, C_{r}$ with $P \cdot C_{i}=0$. Then the divisor $C_{1}+\ldots+C_{r}$ has negative definite intersection matrix thanks to the index theorem. Conversely, given a reduced divisor $C_{1}+\ldots+C_{r}$ with negative definite intersection matrix, we consider the divisor

$$
D \stackrel{\text { def }}{=} H+k\left(C_{1}+\ldots+C_{r}\right)
$$

where $H$ is a fixed ample divisor and $k$ a positive integer. This divisor is big, and for $k \gg 0$ the negative part of its Zariski decomposition will have $C_{1} \cup \ldots \cup C_{r}$ as its support. (The latter fact can for instance be seen from the computation of the Zariski decomposition according to Bau2.)

It will be useful to introduce two abbreviations: If $D$ is a big divisor on a surface, then $\operatorname{Null}(D)$ will denote the set of irreducible curves $C$ such that $D \cdot C=0$. If $D=P_{D}+N_{D}$ is the Zariski decomposition of $D$, with nef part $P_{D}$ and negative part $N_{D}$, then $\operatorname{Neg}(D)$ will denote the set of components of $N_{D}$. In this notation, the Zariski chamber associated with a big and nef divisor $P$ is by definition

$$
\Sigma_{P}=\left\{D \in \operatorname{Big}(X) \mid \operatorname{Neg}(D)=\operatorname{Null}\left(P_{D}\right)\right\} .
$$

Weyl chambers. Let now $X$ be a K3 surface. Apart from the Zariski chamber decomposition, there is a second natural decomposition of the big cone: the decomposition into Weyl chambers. Consider the set $\mathcal{R}(X)$ of irreducible $(-2)$-curves (smooth rational curves of self-intersection -2 ) on $X$, also referred to as the set of simple roots on $X$. Via the intersection product, each of the curves $C \in \mathcal{R}(X)$ defines a hyperplane $C^{\perp}=\{D \mid D \cdot C=0\}$ in $\operatorname{NS}_{\mathbb{R}}(X)$. The connected components of the complement

$$
\operatorname{Big}(X) \backslash \bigcup_{C \in \mathcal{R}(X)} C^{\perp}
$$

yield a decomposition of (a dense open subset of) the big cone into subcones, the simple Weyl chambers. The term simple relates here to the fact that classically one considers not only simple roots, i.e, irreducible (-2)-curves, but roots, i.e., ( -2$)$ classes; taking chambers with respect to $(-2)$-classes leads in general to a finer 
decomposition, whose sets are called Weyl chambers. Also note that classically one considers instead of the big cone $\operatorname{Big}(X)$ the positive cone $C^{+}(X)$, i.e., the cone of divisors $D$ with $D^{2}>0$ and $D \cdot H>0$ for some ample $H$. This cone, which is a subcone of the big cone, enjoys the advantage of being invariant under the Weyl group. In our situation, however, when comparing with the Zariski chamber decomposition, it is more natural to work in the big cone.

Comparing decompositions. Our first result compares the two decompositions of $\operatorname{Big}(X)$. Proposition 3.9 of [BKS] asserts that the Zariski chamber decomposition coincides on K3 surfaces with the Weyl chamber decomposition, but its proof is erroneous. Instead, the two decompositions may differ, and the following result gives the precise condition when this happens.

Theorem 1.2. Let $X$ be a K3 surface. Then the following conditions are equivalent.

(i) The interiors of the Zariski chambers coincide with the simple Weyl chambers.

(ii) There is no pair of (-2)-curves $C_{1}, C_{2} \subset X$ such that

$$
C_{1} \cdot C_{2}=1 .
$$

There exist K3 surfaces such that (i) and (ii) hold, and there exist K3 surfaces where these conditions do not hold.

The equivalence of (i) and (ii) is a consequence of Propositions 2.1 and 2.2 below, which give criteria for mutual inclusions of Zariski and Weyl chambers. We thought, however, that it might be useful to provide a quick direct argument for this basic result right away.

Proof. Suppose first that condition (ii) does not hold, i.e., that there is a pair of (-2)-curves $C_{1}, C_{2}$ with $C_{1} \cdot C_{2}=1$. Choose an ample divisor $H$ and consider the big divisor

$$
D=H+a_{1} C_{1}+a_{2} C_{2}
$$

for positive rational numbers $a_{i}$. Its Zariski decomposition is of the form

$$
D=\left(H+x_{1} C_{1}+x_{2} C_{2}\right)+\left(b_{1} C_{1}+b_{2} C_{2}\right),
$$

where $x_{i} \leqslant a_{i}$ and $b_{i} \leqslant a_{i}$ are non-negative rational numbers satisfying $x_{i}+b_{i}=a_{i}$ and

$$
\left(H+x_{1} C_{1}+x_{2} C_{2}\right) \cdot C_{i}=0
$$

for $i=1,2$. We claim that we can choose the numbers $a_{1}, a_{2}$ in such a way that

$$
\operatorname{Neg}(D)=\left\{C_{1}, C_{2}\right\} \text { and } D \cdot C_{1}>0, D \cdot C_{2}<0 .
$$

Granting (*) for a moment, we consider the big divisor

$$
D^{\prime}=H+k\left(C_{1}+C_{2}\right)
$$

for $k>0$. Thanks to the equality $C_{1} \cdot C_{2}=1$ we have $D^{\prime} \cdot C_{1}<0$ and $D^{\prime} \cdot C_{2}<0$ for $k \gg 0$, and hence $\operatorname{Neg}\left(D^{\prime}\right)=\left\{C_{1}, C_{2}\right\}$ for $k \gg 0$. So we see that $D$ and $D^{\prime}$ lie in the same Zariski chamber, whereas they lie in different simple Weyl chambers. 
Turning to the proof of $(*)$, note first that the $x_{i}$ are independent of the $a_{i}$, since they are uniquely determined by the system of linear equations (1.2.1), whose coefficient matrix is negative definite. Keeping this in mind, we see from the equations

$$
\begin{aligned}
& D \cdot C_{1}=N \cdot C_{1}=\left(b_{1} C_{1}+b_{2} C_{2}\right) C_{1}=-2 b_{1}+b_{2} \\
& D \cdot C_{2}=N \cdot C_{2}=\left(b_{1} C_{1}+b_{2} C_{2}\right) C_{2}=b_{1}-2 b_{2}
\end{aligned}
$$

that $(*)$ will be fulfilled if we take

$$
a_{1}=x_{1}+1 \text { and } a_{2}=x_{2}+3 .
$$

Suppose now that condition (ii) holds. Let $D$ be a big divisor that does not lie on the boundary of any Zariski chamber. It is enough to show that if $C$ is any (-2)-curve, then we have $D \cdot C<0$ if $C \in \operatorname{Neg}(D)$, and $D \cdot C>0$ otherwise. Let $D=P+N$ be the Zariski decomposition. Then $N=\sum b_{i} C_{i}$ with positive rational numbers $b_{i}$ and $(-2)$-curves $C_{i}$. Thanks to negative definiteness and condition (ii), we have $C_{i} \cdot C_{j}=0$ for $i \neq j$. Therefore if $C=C_{1}$, say, then

$$
D \cdot C=N \cdot C=b_{1} C_{1}^{2}=-2 b_{1}<0 .
$$

If $C \notin \operatorname{Neg}(D)$, then clearly $D \cdot C \geqslant 0$. But in fact we have $D \cdot C>0$, since otherwise $C \in \operatorname{Null}(P)$, which by [BKS, Prop. 1.7] would imply that $D$ lies on the boundary of a Zariski chamber.

Finally, we wish to show that there are cases where (i) and (ii) hold and cases where these conditions do not hold. First, there are smooth quartic surfaces in $\mathbb{P}^{3}$ that contain a pair of intersecting lines (see Sect. 3), and hence (ii) does not hold on such surfaces. Secondly, in order to get examples where (ii) does hold, one could take K3 surfaces of Picard number one - there are no $(-2)$-curves at all on such surfaces, so that (ii) is trivially satisfied. More substantially, there are examples of K3 surfaces of Picard number three containing three (-2)-curves, where no two of them intersect with intersection number one (see Proposition 3.4).

We just saw that the Zariski chamber decomposition may differ from the decomposition into simple Weyl chambers. By contrast, our next result shows that, somewhat surprisingly, the number of Zariski chambers always equals the number of simple Weyl chambers.

Theorem 1.3. Let $X$ be a K3 surface. Then there is a canonical bijection between the set of Zariski chambers in $\operatorname{Big}(X)$ and the set of simple Weyl chambers in $\operatorname{Big}(X)$.

Note that the number of chambers may well be infinite, as the number of (-2)curves may be infinite (cf. [Kov, Remark 7.2]).

Proof. As before, denote by $\mathcal{R}(X)$ the set of simple roots, i.e., the set of $(-2)$-curves on $X$. The set of simple Weyl chambers on $X$ is in bijective correspondence with the set

$$
\mathcal{W}(X)=\left\{\begin{array}{l}
\text { subsets } \mathcal{S} \subset \mathcal{R}(X) \text { such that there is a divisor } D \in \operatorname{Big}(X) \\
\text { with } D \cdot C<0 \text { if } C \in \mathcal{S} \text { and } D \cdot C>0 \text { if } C \in \mathcal{R}(X) \backslash \mathcal{S}
\end{array}\right\},
$$

whereas, thanks to Lemma 1.1, the set of Zariski chambers on $X$ is in bijective correspondence with the set

$$
\mathcal{Z}(X)=\left\{\begin{array}{l}
\text { finite subsets } \mathcal{S} \subset \mathcal{R}(X) \text { whose intersection matrix } \\
\text { is negative definite }
\end{array}\right\} \cup \varnothing .
$$


(In both cases, the empty set $\varnothing$ corresponds to the nef chamber.) We will show that $\mathcal{W}(X)=\mathcal{Z}(X)$. To see this, consider first a non-empty set $\mathcal{S}=\left\{C_{1}, \ldots, C_{r}\right\} \in$ $\mathcal{Z}(X)$, and fix an ample divisor $H$. Then for any non-negative rational numbers $a_{i}$, the divisor

$$
D=H+\sum_{i} a_{i} C_{i}
$$

is big. We claim that we can choose the $a_{i}$ in such a way that

$$
D \cdot C_{i}<0 \quad \text { for all } i \text {. }
$$

Since clearly $D \cdot C>0$ for all curves $C$ different from the $C_{i}$, it follows then that the set $\mathcal{S}$ is contained in $\mathcal{W}(X)$. To prove (1.3.2), consider the following system of linear equations for the $a_{i}$,

$$
D \cdot C_{j}=H \cdot C_{j}+\sum_{i=1}^{r} a_{i} C_{i} \cdot C_{j}=-1 \quad \text { for } j=1, \ldots, r .
$$

Its coefficient matrix is negative definite and has non-negative entries outside of the diagonal. An elementary result (see Lemma A.1) implies then that all entries of its inverse matrix are $\leqslant 0$. Therefore the solutions $a_{i}$ are non-negative, and we are done.

Conversely, consider a non-empty set $\mathcal{S} \in \mathcal{W}(X)$. The existence of a big divisor $D$ with $D \cdot C<0$ for $C \in \mathcal{S}$ implies that $\mathcal{S}$ is a finite set $\left\{C_{1}, \ldots, C_{r}\right\}$ and that the intersection matrix of $C_{1}, \ldots, C_{r}$ is negative definite (because the negative part of the Zariski decomposition of $D$ must contain the curves $C_{i}$ ). Therefore $\mathcal{S} \in \mathcal{Z}(X)$.

\section{Inclusions of chambers}

We now give a more detailed description of the mutual inclusions of Weyl and Zariski chambers. We continue to use the notation $\mathcal{Z}(X)$ from (1.3.1) for the set that consists of all sets of $(-2)$-curves whose intersection matrix is negative definite. For $\mathcal{S} \in \mathcal{Z}(X)$, we will write $Z_{\mathcal{S}}$ for the Zariski chamber supported by $\mathcal{S}$, and $W_{\mathcal{S}}$ for the simple Weyl chamber defined by $\mathcal{S}$. (In other words, $Z_{\mathcal{S}}$ consists of the big divisors whose negative part has support $\bigcup \mathcal{S}$, and $W_{\mathcal{S}}$ consists of the big divisors that have negative intersection with the curves in $\mathcal{S}$ and positive intersection with all other (-2)-curves.)

The following two propositions yield Theorem 2 from the introduction. As mentioned before, this result implies in particular the equivalence assertion in Theorem 1 .

Proposition 2.1. Let $X$ be a $K 3$ surface, and let $\mathcal{S} \in \mathcal{Z}(X)$. We have

$$
W_{\mathcal{S}} \subset Z_{\mathcal{S}}
$$

if and only if the following condition holds: If $C^{\prime}$ is a curve with $C^{\prime} \notin \mathcal{S}$ such that the intersection matrix of the set $\mathcal{S} \cup\left\{C^{\prime}\right\}$ is negative definite, then $C^{\prime} \cdot C=0$ for all $C \in \mathcal{S}$.

Proof. Suppose that the stated condition holds. We need to show that one has $\operatorname{Neg}(D)=S$ for every divisor $D \in W_{S}$. We may certainly assume $\mathcal{S} \neq \varnothing$. Let 
then $\mathcal{S}=\left\{C_{1}, \ldots, C_{r}\right\}$ and $D \in W_{\mathcal{S}}$, so that the inequality $D \cdot C_{i}<0$ holds for $i=1, \ldots, r$. In the Zariski decomposition $D=P_{D}+N_{D}$ we have $P_{D} \cdot C_{i} \geqslant 0$, and hence $N_{D} \cdot C_{i}<0$ for all $i$. Therefore we obtain in any event the inclusion $\mathcal{S} \subset \operatorname{Neg}(D)$.

To see the converse inclusion $\operatorname{Neg}(D) \subset \mathcal{S}$, assume by way of contradiction that there is a curve $C^{\prime} \in \operatorname{Neg}(D)$ with $C^{\prime} \notin \mathcal{S}$. From our condition we get $C_{i} \cdot C^{\prime}=0$ for all $i=1, \ldots, r$. Let now $\bigcup_{j=1}^{s} C_{j}^{\prime}$ be the connected component in $\operatorname{Neg}(D)$ containing $C^{\prime}$. Then none of the curves $C_{j}^{\prime}$ can be contained in $S$, and therefore

$$
0 \leqslant D \cdot C_{j}^{\prime}=N_{D} \cdot C_{j}^{\prime}=\sum_{i=1}^{s} a_{i}^{\prime} C_{i}^{\prime} \cdot C_{j}^{\prime}
$$

for $j=1, \ldots, s$. Then Lemma A.1 implies that all $a_{i}^{\prime}$ must be $\leqslant 0$. But this is a contradiction with the fact that $N_{D}$ is effective. Thus $\operatorname{Neg}(D)=\mathcal{S}$, which means that $D \in Z_{\mathcal{S}}$.

To proof the other direction we show: If there is a curve $C^{\prime} \notin \mathcal{S}$ such that $\mathcal{S} \cup\left\{C^{\prime}\right\}$ has negative definite intersection matrix and if there is a curve $C \in \mathcal{S}$ such that $C \cdot C^{\prime}=1$, then there is a divisor $D$ with $D \in W_{\mathcal{S}}$ but $D \notin Z_{\mathcal{S}}$. Assume that $C^{\prime}$ is such a curve. Then there is by Lemma 1.1]a big divisor $B$ with $\operatorname{Neg}(B)=\mathcal{S} \cup\left\{C^{\prime}\right\}$. We can write its Zariski decomposition as

$$
B=P_{B}+N_{B}=P_{B}+b^{\prime} C^{\prime}+\sum_{i=1}^{r} b_{i} C_{i},
$$

where $b^{\prime}$ and $b_{1}, \ldots, b_{r}$ are positive rational numbers. We claim now that we can find positive rational numbers $c^{\prime}$ and $c_{1}, \ldots, c_{r}$ such that the divisor

$$
D=P_{B}+c^{\prime} C^{\prime}+\sum_{i=1}^{r} c_{i} C_{i}
$$

satisfies

$$
D \cdot C^{\prime}>0 \text { and } D \cdot C_{i}<0 \text { for } i=1, \ldots, r .
$$

These inequalities then tell us that $D \in W_{S}$, but $D \notin Z_{S}$.

To prove the existence of $D$, we solve first the system of inequalities

$$
\begin{aligned}
& D \cdot C^{\prime}=N_{D} \cdot C^{\prime}<0 \\
& D \cdot C_{1}=N_{D} \cdot C_{1}<0 \\
& D \cdot C_{r}=N_{D} \cdot C_{r}<0 .
\end{aligned}
$$

for the variables $c^{\prime}, c_{1}, \ldots, c_{r}$. Lemma A.1 guarantees that the solutions are positive. We claim finally that upon replacing $c^{\prime}$ with $c^{\prime}=\frac{1}{4} \min c_{i}$, the desired inequalities (2.1.1) hold. This latter fact follows from a case-by-case analysis using the fact that the connected components of $\operatorname{Neg}(D)$ are A-D-E curves (see Figure 1 for the possible configurations of their components). We omit the details.

Proposition 2.2. Let $X$ be a $K 3$ surface, and let $\mathcal{S} \in \mathcal{Z}(X)$. We have

$$
\stackrel{\circ}{Z}_{\mathcal{S}} \subset W_{\mathcal{S}}
$$

if and only if $C_{1} \cdot C_{2} \neq 1$ for all curves $C_{1}, C_{2} \subset S$. 
Proof. We show first that if there are two curves $C_{i}, C_{j} \in \mathcal{S}$ with $C_{i} \cdot C_{j}=1$, then there is a big divisor $E$, which belongs to $\stackrel{\circ}{Z}_{\mathcal{S}}$ but not to $W_{\mathcal{S}}$. Setting $\mathcal{S}=$ $\left\{C_{1}, \ldots, C_{r}\right\}$, assume that $C_{1}$ and $C_{2}$ are such curves. Choose an ample divisor $H$, and consider the big divisor

$$
D=H+\sum_{i=1}^{r} a_{i} C_{i}
$$

where $a_{1}, \ldots, a_{r}$ are positive rational numbers. Its Zariski decomposition is of the form

$$
D=\underbrace{H+\sum_{i=1}^{r} a_{i}^{*} C_{i}}_{P_{D}}+\underbrace{\sum_{i=1}^{r}\left(a_{i}-a_{i}^{*}\right) C_{i}}_{N_{D}}
$$

where the coefficients $a_{i}^{*}$ satisfy

$$
0=P_{D} \cdot C_{i}=H \cdot C_{i}+\sum_{j=1}^{r} a_{j}^{*} C_{j} \cdot C_{i} \quad \text { for } i=1, \ldots, r .
$$

Choosing the $a_{i}$ large enough, we may assume $0<a_{i}^{*}<a_{i}$ (see [Bau2]). Consider the divisor

$$
D^{*}=H+a_{1}^{*} C_{1}+\sum_{i=2}^{r} a_{i} C_{i}=P_{D}+\sum_{i=2}^{r}\left(a_{i}-a_{i}^{*}\right) C_{i} .
$$

Then we have $P_{D^{*}}=P_{D}$, so $\operatorname{Null}\left(P_{D^{*}}\right)=\operatorname{Null}\left(P_{D}\right)$, but

$$
\operatorname{Neg}\left(D^{*}\right)=\left\{C_{2}, \ldots, C_{r}\right\} \subsetneq\left\{C_{1}, \ldots, C_{r}\right\}=\operatorname{Neg}(D) .
$$

Therefore, by [BKS, Prop. 1.7], the divisor $D^{*}$ lies on the boundary of the Zariski chamber $\Sigma_{P_{D}}$. We have

$$
D^{*} \cdot C_{1}=P_{D} \cdot C_{1}+\sum_{i=2}^{r}\left(a_{i}-a_{i}^{*}\right) C_{i} \cdot C_{1} \geqslant\left(a_{2}-a_{2}^{*}\right) C_{2} \cdot C_{1}=a_{2}-a_{2}^{*}>0 .
$$

There exists an $\varepsilon>0$, such that $\left(D^{*}+\varepsilon C_{1}\right) \cdot C_{1}>0$, and the divisor $D^{*}+\varepsilon C_{1}$ lies in the interior of $\Sigma_{P_{D}}$ by [BKS, Prop. 1.8]. Setting $E=D^{*}+\varepsilon C_{1}$ we are done.

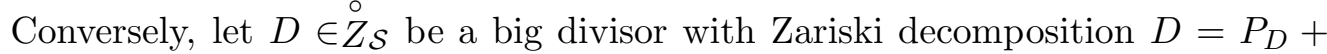
$N_{D}$. Then $N_{D}=\sum_{i=1}^{r} a_{i} C_{i}$ with positive rational numbers $a_{i}$ and $(-2)$-curves $C_{i}$. Thanks to negative definiteness and the condition in the proposition we have $C_{i} \cdot C_{j}=0$ for $i \neq j$. Therefore:

$$
D \cdot C_{i}=N_{D} \cdot C_{i}=a_{i} C_{i}^{2}=-2 a_{i}<0 \quad \text { for } i=1, \ldots, r .
$$

For any (-2)-curve $C$ different from the $C_{i}$, we clearly have $D \cdot C \geqslant 0$. We claim that $D \cdot C>0$. In fact, if we had $D \cdot C=0$, then $P_{D} \cdot C=0$. So $C \in \operatorname{Null}(P)$, but $C \notin \operatorname{Neg}(D)$, and then, again by [BKS, Prop. 1.7], $D$ would lie on the boundary of the chamber $Z_{S}$. 


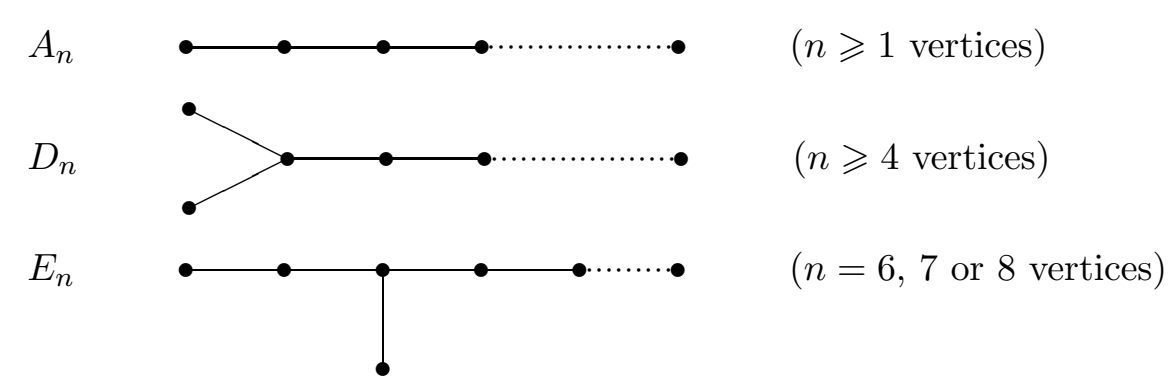

Figure 1: The Dynkin diagrams corresponding to A-D-E curves on a K3 surface.

\section{Examples}

K3 surfaces with differing decompositions. We analyze now in detail examples of K3 surfaces where the two chamber decompositions differ. Specifically, we consider smooth quartic surfaces in $\mathbb{P}^{3}$ containing a hyperplane section that decomposes into two lines and an irreducible conic. It is easy to see - for instance as in Bau1, Lemma 2.2b] - that such surfaces exist. Fix such a quartic surface $X$, and let $L_{1}, L_{2}, C$ be two lines and an irreducible conic such that $L_{1}+L_{2}+C$ is a hyperplane section of $X$. In the Néron-Severi vector space $\operatorname{NS}_{\mathbb{R}}(X)$ we consider the subspace

$$
V \stackrel{\text { def }}{=}\left\langle L_{1}, L_{2}, C\right\rangle \subset \mathrm{NS}_{\mathbb{R}}(X)
$$

spanned by the classes of $L_{1}, L_{2}$, and $C$. The intersection form is given on $V$ by the matrix

$$
\left(\begin{array}{ccc}
-2 & 1 & 2 \\
1 & -2 & 2 \\
2 & 2 & -2
\end{array}\right) .
$$

We now show:

Proposition 3.1. (i) The intersection $\mathcal{C} \stackrel{\text { def }}{=} \operatorname{Big}(X) \cap V$ is the interior of the cone generated by the classes of $L_{1}, L_{2}$, and $C$.

(ii) The curves $L_{1}, L_{2}$, and $C$ are the only $(-2)$-curves in $V$.

(iii) The intersection $\operatorname{Nef}(X) \cap V$ consists of the classes $a C+b_{1} L_{1}+b_{2} L_{2}$ such that the real numbers $a, b_{1}, b_{2}$ satisfy the inequalities

$$
b_{1}+b_{2} \geqslant a, \quad 2 a+b_{2} \geqslant 2 b_{1}, \quad 2 a+b_{1} \geqslant 2 b_{2} .
$$

(iv) The cone $\mathcal{C}$ decomposes into five Zariski chambers and into five simple Weyl chambers. These two decompositions do not coincide.

Proof. (i) $\operatorname{As} \operatorname{Big}(X)$ is the closure of the effective cone, the issue is to see that if a numerical class $D=a C+b_{1} L_{1}+b_{2} L_{2} \in V$ is effective, then all coefficients $a, b_{1}, b_{2}$ are non-negative. Note that if we had $V=\mathrm{NS}_{\mathbb{R}}(X)$, then this would follow from [Kov, Theorem 6.1]. In our situation it can be seen as follows: One checks first that the divisor $C+2 L_{1}+2 L_{2}$ is nef. From the fact that its intersection with $D$ must be non-negative we get then that $a \geqslant 0$. Proceeding in the same way with the nef divisors $C+L_{1}$ and $C+L_{2}$ we get $b_{2} \geqslant 0$ and $b_{1} \geqslant 0$ respectively. 

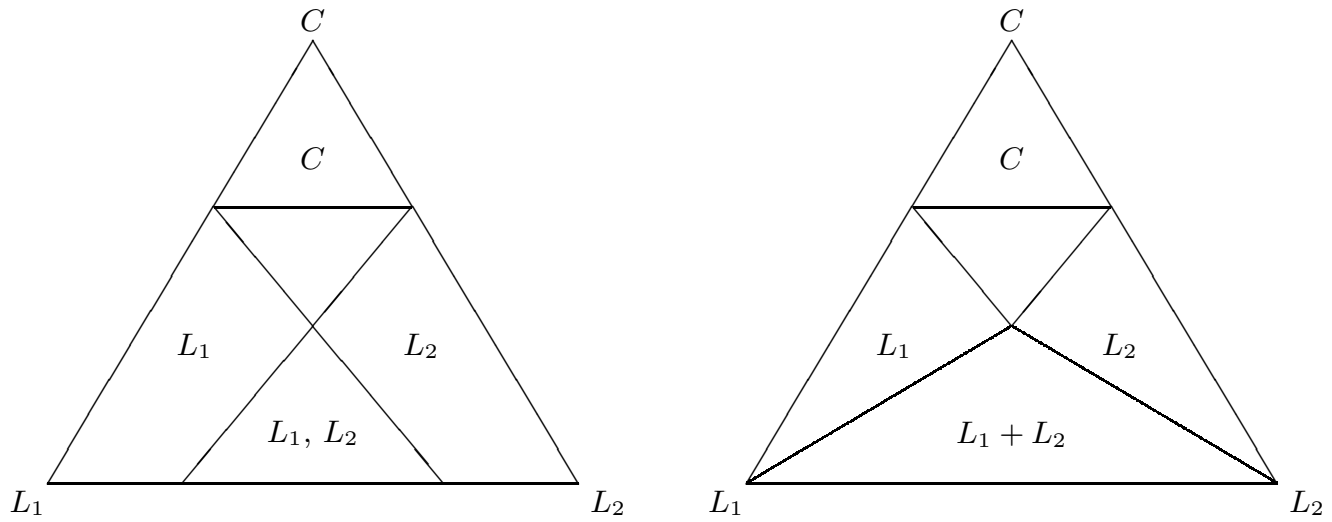

Figure 2: The Weyl chamber decomposition (left picture) and the Zariski chamber decomposition (right picture) of the cone $\mathcal{C}$ on the quartic surfaces from Proposition 3.1 The pictures show the intersection of the (3-dimensional) cone $\mathcal{C}$ with the hyperplane in $V$ passing through the classes of $L_{1}, L_{2}$, and $C$. The labels in the chambers indicate in the left picture the curves that are intersected negatively and in the right picture the support of the Zariski chambers. The unlabelled chamber in the center is the nef chamber, i.e., the chamber whose interior is the ample cone and whose closure is the nef cone.

(ii) Suppose that $F$ is a (-2)-curve in $V$. Then by (i) the numerical class of $F$ lies in the closure of $\mathcal{C}$. Writing $F=a C+b_{1} L_{1}+b_{2} L_{2}$ for this class, we have

$$
\left(a C+b_{1} L_{1}+b_{2} L_{2}\right) F=F^{2}=-2<0 .
$$

As $F$ is irreducible and the divisor in brackets is effective, this can only happen if $F$ is one of the curves $C, L_{1}, L_{2}$.

(iii) A class $a C+b_{1} L_{1}+b_{2} L_{2}$ is nef if and only if it meets the curves $C, L_{1}, L_{2}$ non-negatively. These three conditions yield the asserted inequalities.

(iv) The intersection matrix (3.0.1) has exactly four negative definite prinicipal submatrices, corresponding to the four divisors

$$
L_{1}, L_{2}, L_{1}+L_{2}, C \text {. }
$$

Therefore, by Lemma 1.1, there are exactly five Zariski chambers supported on the curves $L_{1}, L_{2}, C$, and hence by Theorem 1.3 also exactly five Weyl chambers. The fact that the decompositions do not coincide is of course a consequence of Theorem 1.2. Alternatively, it can be verified directly by computing the chambers from the shape of $\operatorname{Nef}(X) \cap V$, i.e., using the inequalities given in (iii).

Remark 3.2. The two decompositions of $\mathcal{C}$ are shown in Figure 2, Using Theorem 2 we get also information about the chamber decomposition of the cone $\operatorname{Big}(X)$ (which might be bigger than $\mathcal{C}$ ). In particular, we have the following inclusions and noninclusions of chambers on $X$ :

(i) $\stackrel{\circ}{Z}_{\varnothing}=W_{\varnothing}$, as always,

(ii) $\stackrel{\circ}{Z}_{\left\{L_{1}\right\}} \subsetneq W_{\left\{L_{1}\right\}}$ and $\stackrel{\circ}{Z}_{\left\{L_{2}\right\}} \subsetneq W_{\left\{L_{2}\right\}}$,

(iii) $\stackrel{\circ}{Z}_{\{C\}} \subset W_{\{C\}}$,

(iv) $\stackrel{\circ}{Z}_{\{C\}}=W_{\{C\}}$, if and only if there is no $(-2)$-curve $C^{\prime} \subset X$ with $C^{\prime} \cdot C=1$. 
(v) $\stackrel{\circ}{Z}_{\left\{L_{1}, L_{2}\right\}} \not \subset W_{\left\{L_{1}, L_{2}\right\}}$

(vi) $W_{\left\{L_{1}, L_{2}\right\}} \subset Z_{\left\{L_{1}, L_{2}\right\}}$, if any only if there is no (-2)-curve $C^{\prime} \subset X$ such that $C^{\prime} \cdot L_{1}=0$ and $C^{\prime} \cdot L_{2}=1$ or conversely.

The situation becomes particulary transparent when one has quartics as above whose Picard number is exactly three. We will show that that surfaces with this property exist:

Proposition 3.3. There exist smooth quartic surfaces $X \subset \mathbb{P}^{3}$ of Picard number three having a hyperplane section of the form $L_{1}+L_{2}+C$ such that $L_{1}$ and $L_{2}$ are lines and $C$ is a smooth conic.

On such surfaces one has $V=\mathrm{NS}_{\mathbb{R}}(X)$, and then the conditions in (iv) and (vi) are fulfilled by Proposition 3.1. Therefore, in that case

- $\stackrel{\circ}{Z}_{\left\{L_{1}\right\}} \subsetneq W_{\left\{L_{1}\right\}}$ and $\stackrel{\circ}{Z}_{\left\{L_{2}\right\}} \subsetneq W_{\left\{L_{2}\right\}}$,

- $\stackrel{\circ}{Z}_{\{C\}}=W_{\{C\}}$,

- $W_{\left\{L_{1}, L_{2}\right\}} \subsetneq Z_{\left\{L_{1}, L_{2}\right\}}$.

The picture in Figure 2 describes then the whole big cone of $X$.

Proof of Proposition 3.3. Consider the K3 lattice

$$
\Lambda_{K_{3}}=U^{3} \oplus\left(-E_{8}\right)^{2}
$$

where $U$ is the lattice $\mathbb{Z}^{2}$ with the bilinear form given by the matrix

$$
\left(\begin{array}{ll}
0 & 1 \\
1 & 0
\end{array}\right)
$$

and $-E_{8}$ is the lattice $\mathbb{Z}^{8}$ with the bilinear form given by

$$
\left(\begin{array}{cccccccc}
-2 & 1 & 0 & 0 & 0 & 0 & 0 & 0 \\
1 & -2 & 1 & 0 & 0 & 0 & 0 & 0 \\
0 & 1 & -2 & 1 & 0 & 0 & 0 & 0 \\
0 & 0 & 1 & -2 & 1 & 0 & 0 & 0 \\
0 & 0 & 0 & 1 & -2 & 1 & 0 & 1 \\
0 & 0 & 0 & 0 & 1 & -2 & 1 & 0 \\
0 & 0 & 0 & 0 & 0 & 1 & -2 & 0 \\
0 & 0 & 0 & 0 & 1 & 0 & 0 & -2
\end{array}\right)
$$

For any $\mathrm{K} 3$ surface $X$, there is an isomorphism $\sigma: H^{2}(X, \mathbb{Z}) \rightarrow \Lambda_{K 3}$. The 21dimensional period space

$$
\Omega=\left\{\mathbb{C} \cdot x \in \mathbb{P}\left(\Lambda_{K_{3}} \otimes \mathbb{C}\right) \mid\langle x, x\rangle=0,\langle x, \bar{x}\rangle>0\right\}
$$

is a fine moduli space for marked $\mathrm{K} 3$ surfaces, i.e., for pairs $(X, \sigma)$ consisting of a $\mathrm{K} 3$ surface $X$ and an isormorphism $\sigma: H^{2}(X, \mathbb{Z}) \rightarrow \Lambda_{K 3}$ (see e.g. [BPV] for the facts mentioned here).

Let now $X_{0} \subset \mathbb{P}^{3}$ be a smooth quartic surface admitting a hyperplane section of the form $L_{1}+L_{2}+C$ as considered above, and let $\lambda_{1}, \lambda_{2}, \gamma \in \Lambda_{K 3}$ be the lattice vectors corresponding to the classes of $L_{1}, L_{2}, C$ under a fixed isomorphism 
$H^{2}(X, \mathbb{Z}) \stackrel{\sim}{\longrightarrow} \Lambda_{K 3}$. The point $x_{0} \in \Omega$ corresponding to $X_{0}$ is contained in the intersection

$$
\Omega^{\prime}=\Omega \cap \lambda_{1}^{\perp} \cap \lambda_{2}^{\perp} \cap \gamma^{\perp} .
$$

Consider now a small deformation $X$ of $X_{0}$ corresponding to a point $x \in \Omega^{\prime}$ close to $x_{0}$. As $\Lambda_{K 3}$ is a countable set, the generic such $x$ is not contained in any intersection

$$
\Omega^{\prime} \cap \alpha^{\perp}
$$

where $\alpha \in \Lambda_{K_{3}}$ is a lattice vector different from $\lambda_{1}, \lambda_{2}, \gamma$. But this implies that the Picard group of $X$ is generated over $\mathbb{Q}$ precisely by the classes of $L_{1}, L_{2}$ and $C$. The Riemann-Roch theorem implies that these $(-2)$-classes are on the deformed surface still represented by effective divisors. Therefore, when $x$ is close enough to $x_{0}$, they are still represented by irreducible curves.

K3 surfaces with coinciding decompositions. If a K3 surface does not contain any $(-2)$-curves at all - for instance if its Picard group is one-dimensional - then of course the Zariski chamber decomposition coincides with the Weyl chamber decomposition. More substantial examples are given by the following proposition. The double covering construction in its proof was suggested to us by T. Szemberg.

Proposition 3.4. There exist K3 surfaces $X$ of Picard number three that contain three (-2)-curves $F_{1}, F_{2}, C$ such that

$$
F_{1} \cdot F_{2}=0, \quad F_{1} \cdot C=F_{2} \cdot C=2,
$$

and such that there are no other $(-2)$ curves on $X$.

The decomposition of the big cone of $X$ into Zariski chambers is the same as the decomposition into simple Weyl chambers. It consists of five chambers.

Proof. Let $B \subset \mathbb{P}^{2}$ be a sextic curve that has two ordinary double points $x_{1}$ and $x_{2}$ and no other singularities, and consider the blow-up $f: Y=\mathrm{Bl}_{x_{1}, x_{2}}\left(\mathbb{P}^{2}\right) \rightarrow \mathbb{P}^{2}$ at these two points. On the blow-up let $B^{\prime}$ be the proper transform of $B$, i.e.,

$$
B^{\prime}=f^{*} B-2 E_{1}-2 E_{2},
$$

where $E_{1}$ and $E_{1}$ are the exceptional divisors over $x_{1}$ and $x_{2}$ respectively. The line bundle $\mathcal{O}_{Y}\left(B^{\prime}\right)$ is then 2-divisible, so that we can consider the double covering

$$
g: X \rightarrow Y
$$

that is ramified over $B^{\prime}$. As $K_{X}=g^{*}\left(K_{Y}+\frac{1}{2} B^{\prime}\right)=0$ and $h^{1}\left(\mathcal{O}_{X}\right)=0$ (cf. [BPV, Sect. 22]), the surface $X$ is a K3 surface. One checks that the pullbacks

$$
F_{1}=g^{*} E_{1}, \quad F_{2}=g^{*} E_{2}, \quad C=g^{*}\left(H-E_{1}-E_{2}\right)
$$

are $(-2)$-curves on $X$. These curves generate a subspace of the Néron-Severi vector space of dimension three, on which the intersection form is given by the matrix

$$
\left(\begin{array}{ccc}
-2 & 0 & 2 \\
0 & -2 & 2 \\
2 & 2 & -2
\end{array}\right)
$$

Arguing as in the proof of Proposition 3.3, i.e., replacing $X$ with a small deformation in the period space, we obtain a K3 surface of Picard number three with curves 
$F_{1}, F_{2}, C$ intersecting as in (3.4.1) that generate the Néron-Severi group over $\mathbb{Q}$. For simplicity, let us denote the deformed surface again by $X$.

We show next that there are no other $(-2)$-curves on $X$. Suppose that $D$ is a $(-2)$-curve on $X$, and write in numerical equivalence

$$
D=a C+b_{1} F_{1}+b_{2} F_{2}
$$

with rational numbers $a, b_{1}, b_{2}$. We can now proceed as in the proof of Proposition 3.1(i) to show that $a \geqslant 0$ and $b_{1}, b_{2} \geqslant 0$. (In fact, intersect $D$ with the nef divisors $C+F_{1}, C+F_{2}$, and $C+F_{1}+F_{2}$.) One sees then as in the proof of Proposition 3.1(ii) that $F_{1}, F_{2}$, and $C$ are the only $(-2)$-curves on $X$. The intersection matrix (3.4.1) has exactly four negative definite principal submatrices, corresponding to the divisors

$$
F_{1}, F_{2}, F_{1}+F_{2}, C .
$$

With Lemma 1.1 we conclude that there are exactly five Zariski chambers, and Theorem 1.2 implies that the chamber decompositions coincide.

\section{Appendix}

The following useful lemma from BKS is used several times in the present paper. While probably well-known, a somewhat technical proof was included in BKS] for lack of a reference. Here we give a much simpler argument, which is inspired by the beginning of the proof of [PR, Theorem 3.2].

Lemma A.1. Let $S$ be a negative definite $(r \times r)$-matrix over the reals such that $s_{i, j} \geqslant 0$ for $i \neq j$. Then all entries of the inverse matrix $S^{-1}$ are $\leqslant 0$.

Proof. It is enough to show that if $a, b \in \mathbb{R}^{r}$ are vectors with $a=S^{-1} b$ such that $b_{i} \leqslant 0$ for all $i$, then $a_{i} \geqslant 0$ for all $i$. To prove this claim, write $a=p-q$, where $p_{i}=\max \left(a_{i}, 0\right)$. Then $p_{i} \geqslant 0, q_{i} \geqslant 0$, and $p_{i} q_{i}=0$ for all $i$. Using these relations along with the hypothesis that $s_{i, j} \geqslant 0$ for $i \neq j$, we get

$$
p^{t} S q=\sum_{i, j} p_{i} s_{i, j} q_{j}=\sum_{i \neq j} p_{i} s_{i, j} q_{j} \geqslant 0
$$

and hence

$$
p^{t} S q-q^{t} S q>0 \quad \text { if } q \neq 0,
$$

because $S$ is negative definite. On the other hand, we have

$$
p^{t} S q-q^{t} S q=(p-q)^{t} S q=a^{t} S q=(S a)^{t} q=b^{t} q \leqslant 0,
$$

so we conclude that $q=0$. This implies that $a_{i} \geqslant 0$ for all $i$, as claimed.

\section{References}

[BPV] Barth, W., Peters, C., Van de Ven, A.: Compact complex surfaces. Ergeb. Math. Grenzgeb. (3) 4, Springer-Verlag, 1984

[Bau1] Bauer, Th.: Seshadri constants of quartic surfaces. Math. Ann. 309, 475-481 (1997)

[Bau2] Bauer, Th.: A simple proof for the existence of Zariski decompositions on surfaces. J. Algebraic Geom. 18, 789-793 (2009) 
[BFN] Bauer, Th., Funke, M., Neumann, S.: Counting Zariski chambers on Del Pezzo surfaces. Journal of Algebra 324, 92-101 (2010)

[BKS] Bauer, Th., Küronya, A., Szemberg, T.: Zariski chambers, volumes, and stable base loci. J. reine angew. Math. 576, 209-233 (2004)

[ELMNP1] L. Ein, R. Lazarsfeld, M. Mustaţă, M. Nakamaye and M. Popa, Asymptotic invariants of line bundles, Pure and Applied Math. Quarterly, A. Borel issue, 1 (2005), no.2, 139-163.

[ELMNP2] L. Ein, R. Lazarsfeld, M. Mustaţă, M. Nakamaye and M. Popa: Restricted volumes and base loci of linear series. Am. J. of Math. 131, 607-651 (2009)

[Kov] Kovács, S.J.: The cone of curves of a K3 surface. Math. Ann. 300, 681-691 (1994)

[Laz] Lazarsfeld, R.: Positivity in algebraic geometry I \& II. Ergebnisse der Mathematik und ihrer Grenzgebiete, vol. 48 \& 49, Springer-Verlag, Berlin, 2004.

[Nak] Nakamaye, M.: Stable base loci of linear series. Math. Ann. 318 (2000), 837-847.

[PR] Panov, D., Ross, J.: Slope stability and exceptional divisors of high genus. Math. Ann. 343, 79-101 (2009)

Thomas Bauer, Fachbereich Mathematik und Informatik, Philipps-Universität Marburg, Hans-Meerwein-Straße, D-35032 Marburg, Germany.

E-mail address: tbauer@mathematik.uni-marburg.de

Michael Funke, Fachbereich Mathematik und Informatik, Philipps-Universität Marburg, Hans-Meerwein-Straße, D-35032 Marburg, Germany.

E-mail address: funke@mathematik.uni-marburg.de 